\title{
Semi-Direct Routing Approach for Mobile IP
}

\author{
Basil Al-Kasasbeh \\ Faculty of Computer Studies \\ Arab Open University (AOU), Riyadh, Kingdom of Saudi Arabia
}

\begin{abstract}
The Mobile IP (MIP) protocol is used to maintain device connectivity while the device is moving between networks through a permanent IP address and temporary care-of-address (CoA). There are two techniques to implement MIP; these are direct and indirect. The indirect is commonly used in the current industry due to its stability while the mobile host (MH) frequently moves from network to another. However, the indirect technique suffers from the problems of delays and enlargement related to the packet size. The direct technique is more sensitive to frequent mobility, yet it required less transformation overhead with stable mobility. Accordingly, to overcome the disadvantages of both techniques, a semi-direct technique is proposed in this paper. The proposed technique is implemented by minimizing the home agent's interference (HA) with a push notification to the correspondent node $(\mathrm{CN})$ that concerns any modification in the moving MH's CoA. The simulation of the proposed technique, the indirect and the direct routing techniques showed the advantages of the semi-direct routing technique over the conventional approaches. The results showed that the semi-direct approach outperformed the conventional approaches in terms of delay and overhead with frequently moved MH.
\end{abstract}

Keywords-Mobile IP; direct routing; indirect routing; care-of address; home agent; foreign agent

\section{INTRODUCTION}

Internet mobility is a crucial aspect of the IP networks that support delivering the data to a moving node [1]. Although different protocols and solutions have been proposed to support mobility, Mobile IP (MIP) protocol is a wellestablished and commonly utilized protocol [2, 3]. Accordingly, it has been extensively studied and extended to support efficient mobility in network architectures [4]. Mobile IP (MIP) is a communication protocol standardized by the Internet Engineering Task Force (IETF). It is developed to enable connected devices to move from one network to another while maintaining connectivity. The MIP enables the routing of IP packets of a moving mobile node (MN) and enables MN to be location independent [5]. Table I presented the set of abbreviations that are required to discuss the MIP [6].

MIP is focused on the mobility of an MN, a device with a network interface that can move from a network to another and send and receive data packets. $\mathrm{MN}$ can communicate with other MN, which in this case is called Correspondent Node $(\mathrm{CN})$. Any node in MIP is identified by three types of addresses based on its location; these are Permanent address (PA), the media access control address, abbreviated as MAC address (MA) and care-of-address (CoA). These addresses can be a source address (SA) or destination address (DA) associated with a transmitted packet. The PA for the $\mathrm{MH}$ is a fixed number assigned by the service provider and allows the $\mathrm{MH}$ to be publicly visible. MA is also a unique and permanent address assigned by the manufacturer to be used for communications within a network segment. The CoA is a temporary address used only when the $\mathrm{MN}$ is outside the $\mathrm{HM}$ [6].

The MIP network architecture consists of essential entities and addressing schemes, as illustrated in Fig. 1. Each network involves the home agent (HA) and the foreign agent (FA) and a set of local MN and possibly visiting MNs that are moved from other networks. For example, in Fig. 1, in network A there is a visiting $\mathrm{MN}$ from network $\mathrm{B}$, a visiting $\mathrm{MH}$ in network $\mathrm{B}$ from network $\mathrm{A}$ and a visiting $\mathrm{MH}$ in network $\mathrm{C}$ from network B.

PA is solely used when the MN is in its HN. On the other hand, when the MN is outside the $\mathrm{HN}$, it is associated with the special CoA. The CoA identifies the current location using an endpoint related to a local tunnel, which leads to the HA. CoA is also responsible for specifying the MN's registration with the HA along with the routing of IP packets through the tunnel. The internet connection is maintained between the stationary and the MN to avoid the negative effects associated with the change of location during the node's mobility. Hence, it keeps the Internet connection in an active mode [7].

TABLE I. DEFINITION OF THE ABBREVIATIONS AND TERMINOLOGY

\begin{tabular}{|l|l|}
\hline Term. (Abb.) & Definition \\
\hline $\begin{array}{l}\text { Mobile Node } \\
\text { (MN) }\end{array}$ & $\begin{array}{l}\text { A device with a network interface that can move from a } \\
\text { network to another }\end{array}$ \\
\hline $\begin{array}{l}\text { Correspondent } \\
\text { Node (CN) }\end{array}$ & A connected device that is communicating with MN \\
\hline $\begin{array}{l}\text { Permanent } \\
\text { Address (PA) }\end{array}$ & The public IP address that is assigned to an MN \\
\hline $\begin{array}{l}\text { MAC Address } \\
\text { (MA) }\end{array}$ & An address that is used as a unique identifier for MN \\
\hline $\begin{array}{l}\text { Care-of-Address } \\
\text { (CoA) }\end{array}$ & $\begin{array}{l}\text { A temporary IP address assigned to a moving MN outside } \\
\text { the HN }\end{array}$ \\
\hline $\begin{array}{l}\text { Source Address } \\
\text { (SA) }\end{array}$ & $\begin{array}{l}\text { An address of the MN from which the transmitted packet } \\
\text { is originated }\end{array}$ \\
\hline $\begin{array}{l}\text { Destination } \\
\text { Address (DA) }\end{array}$ & $\begin{array}{l}\text { An address of the MN to which the transmitted packet is } \\
\text { addressed }\end{array}$ \\
\hline $\begin{array}{l}\text { Home Agent } \\
\text { (HA) }\end{array}$ & $\begin{array}{l}\text { A device that stores information about the locations and } \\
\text { addresses of the devices in the MN }\end{array}$ \\
\hline $\begin{array}{l}\text { Foreign Agent } \\
\text { FA) }\end{array}$ & $\begin{array}{l}\text { A device that stores information about nodes visiting its } \\
\text { network and advertise CoA }\end{array}$ \\
\hline $\begin{array}{l}\text { Home Network } \\
\text { (HN) }\end{array}$ & $\begin{array}{l}\text { A network to which an MN belongs and identified } \\
\text { through its address }\end{array}$ \\
\hline $\begin{array}{l}\text { Foreign } \\
\text { Network (FN) }\end{array}$ & A network MN is locating when away from its HN \\
\hline
\end{tabular}

This work was supported by the Arab Open University, KSA. 


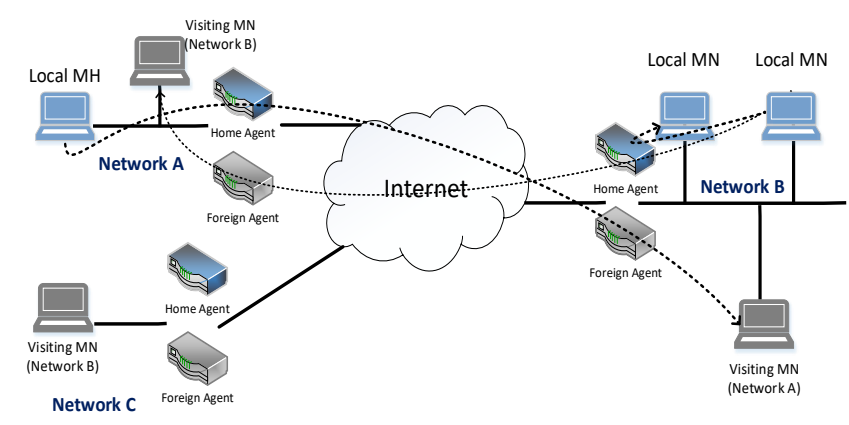

Fig. 1. Mobile IP Architecture.

The data packets transmitted to an MN using MIP are directed into two essential entities: HA and FA. The HA stores the information about the nodes that belong to the home network (HN) through their PA. Additionally, the HA functions as a router at the $\mathrm{HN}$ for the $\mathrm{MNs}$ and ensures the delivery of packets through a tunnelling process to the $\mathrm{MN}$ when the $\mathrm{MN}$ is located away from the $\mathrm{HN}$ by maintaining the location directory [8, 9]. On the other hand, the FA is responsible for storing information about MNs that visiting the network. The FA is also responsible for the advertisement of the CoA of the visiting nodes. FA also functions as a router on the network that offers different services for routing the registered MN. Moreover, the FA delivers and de-tunnels datagrams to the $\mathrm{MN}$ that is tunnelled through the HA. In case there is no FA, the MN has to take the responsibility of addressing and advertising the address by itself [10].

Although HA and FA have predetermined roles in the MIP, these roles change based on the implemented routing technique, indirect or direct. The indirect technique is commonly utilized due to its stability while the $\mathrm{MH}$ is moving from network to another frequently. However, it suffers from the problems of delays and enlargement related to the packet size. The direct approach is more sensitive to frequent mobility, yet it required less transformation overhead with stable mobility.

The direct routing technique, also called host-based mobility protocols [11], enables $\mathrm{CN}$ to send a packet to $\mathrm{MN}$ by using the CoA if the MN located in a visited FN. As illustrated in Fig. 2, the HA is responsible for interacting with a FA of the visiting network to track the mobile station's temporary address (CoA). The $\mathrm{CN}$ sends a request to the HA for the CoA of the MA. Packets are sent from the CN to the MN directly based on the CoA as illustrated in Fig. 2. HA has no roles in packet transmissions, and it just enables the $\mathrm{CN}$ to send packets directly by providing and tracking the CoA of the MN outside the HN. As the MN frequently moving from a network to another, the communication overhead increased as a result of $\mathrm{CoA}$ requesting and packet re-transmission [12, 13].

Indirect routing technique, also called network-based mobility protocols [14], enables a $\mathrm{CN}$ to send a packet to $\mathrm{MN}$ by using the PA through the HA whether an MN is located in its $\mathrm{HN}$ or a visiting an FN. Hence, mobility is completely transparent to its CN. Packets are first forwarded to the HA, as illustrated in Fig. 2. The HA is responsible for interacting with a FA to track the mobile station's temporary address (CoA). HA also identifies the arriving packets addressed to the MNs whose permanent address is associated with that HA, but currently located in an FN. The HA receives these packets and forwards those to an MN based on two steps. The packet is first forwarded to the correspondent FA by using the MN's CoA and are forwarded afterwards from the FA to the MN. The HA addresses the packet by using the CoA so that the network layer can be able to route the packet through to the FN by applying several conventional routing algorithms. The HA encapsulates the original complete packet within a new larger packet, which is addressed and delivered to the MN's CoA. The FA for which the CoA belongs receives and decapsulates the packet and extracts the correspondent's original packet from the larger encapsulating packet $[15,16]$.

The growth with the number of connected and moving MN demands for fast and scalable connections with the shortest routing path and minimum handover latency. The existing techniques suffer from delay and overhead problems. For example, in the host-based protocol, as the MN is transmitted from $\mathrm{FN}$ to another FN, the transmission could be delivered to the wrong node and lead to communication overhead in retransmitting the packets. Similarly, as the MN is transmitted from $\mathrm{FN}$ to another $\mathrm{FN}$ in the network-based protocol, the transmission required a registration processing with overhead communication between the HA and the FA. The gap in the existing techniques embodied in the delay in delivering the transmitted packets and communication overhead with a moving MN. Given the recent advances in software and hardware devices, MNs frequently move with demands to improve delay and overhead and enable intelligent real-time networks [17].

Accordingly, a semi-direct approach is proposed in this paper to reduce the delay and communication overhead with frequently moving MNs. The proposed a semi-direct technique to overcome the problems of both techniques. The semi-directed technique is implemented by minimizing the HA's interference with a push notification to the $\mathrm{CN}$ that concerns any modification in the CoA of moving $\mathrm{MH}$. The proposed technique depends on changing the route of the transmitted packets and changes the HA and FA roles compared to the direct and indirect approach. Accordingly, in the semi-direct technique, the HA of the local network and the FA of the visiting network are responsible for verifying the address known to the $\mathrm{CN}$ and forwarding the data without encapsulation and decapsulation.

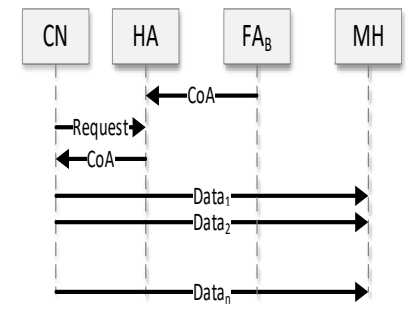

Direct

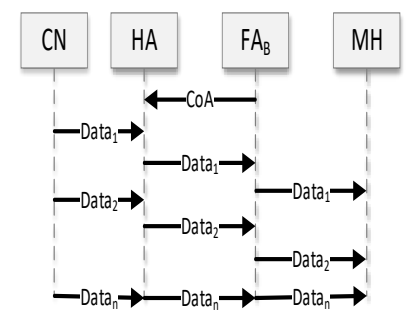

Indirect
Fig. 2. Direct vs Indirect MIP Techniques. 
The rest of this paper is organized as follows: Section 2 gives an overview of the related work that concerns the MIP extensions. In Section 3, the proposed work is presented and discussed in details. Section 4 presents the comparative results of the proposed and the existing techniques, both using analytical and simulation approaches, and finally, Section 5 concludes this paper.

\section{RELATED WORK}

Various mobile IP routing techniques enable users to be moving through various networks during the reception of support sessions and establishment session requests [18]. In the attempt to minimize the delay and overhead, existing MIP developments have taken different directions, these are: 1) anchor distribution over the networks 2) global dynamic home agent, 3) collector-based location tracking, and 4) predict mobility pattern.

\section{A. Anchor Distribution}

The anchor distribution solutions have been developed to eliminate the need for the HA-FA communication overhead and delay with the MN's movement. According to [19] the HA-FA communication overhead can be solved based on "distribution of home agents inside the current Internet topology to reduce distances to end-node". The $\mathrm{MN}$ is bind with the closest anchor. The $\mathrm{CN}$ can request the address from all available anchors using anycast routing and directly communicating with the MN without the HA's inferencing (e.g., anchor). Similar anchor distribution approaches with different topologies and different network architectures were proposed [20, 21]. In [22] a method for enabling the visiting node to implement a temporary agent within the visited mobile network was proposed. Hence, communication is considered intermediate and is accompanied by the Dynamic Home Agent (DHA) to minimize the risk of encountering a single failure point and deliver a flexible system. In particular, the DHA allocates an impermanent home address for the visiting node for reducing the distance between the HA and the visiting node, and in contrast, reducing the handover latency.

The advantage of anchor distribution approach is reducing the overhead and the delay if the anchor is distributed in the network, which leads to another problem of how to deploy the anchors in the network to avoid the extra signalling cost while avoiding interference of the HA.

\section{B. Global Dynamic Home Agent}

In [23] a Global Dynamic Home Agent Discovery (GDHAD) method was proposed, which enables the visiting node to apply the nearest HA within any casting protocol that explores the nearest HA of each node. This method minimizes the cost and delay of the registration process with the original HA. In [24] a different disseminated HA-based method for minimizing the cost and delay of the registration process was proposed. This method relies on a peer-to-peer HA exploration and overlays peer-to-peer networks designed via several home agents. Every home agent contains information regarding other HAs' locations. A mobile station remains to deliver its new location through to the new HA that starts with an exploration procedure to search for the nearest HA to the $\mathrm{MH}$.

Furthermore, the new HA delivers the MH through to the IP addresses, which found the nearest to their HAs, by connecting to any of these HAs. Similarly, in [25] another method to allocate the nearest HA was proposed. The $\mathrm{MH}$ delivers a registration request to the new home agent that preserves the path and historical information about the disseminated HA and MH. Moreover, the new HA searches for the most appropriate one that depends on the history maintained and began with the chosen HA's delegation process. Accordingly, the new HA delivers an acknowledgement through to the HA following the completion of this process. Furthermore, in [26] a mobility management method that can minimize the procedure's cost of a particular communication based on the Markov procedure's use was proposed, which investigates the tracking cost related to the device mobility. A mobile station (MS) grouping and equivalent quadrangular area are applied to minimize the communication overhead and the available stations.

\section{Collector-based Location Tracking}

Using a software-defined network (SDN), each node in the network can be an anchor for the visiting node. Accordingly, if the $\mathrm{MN}$ and the $\mathrm{CN}$ are linked to a different anchor, then the communication problem can be eased if the communication between the controllers is within the same administrative domain [4, 27]. The problems with the SDN is not implemented currently on the Internet. Thus, it cannot be generalized in this form.

\section{Predicting Mobility Pattern}

For predicting the mobility pattern, in [28] a technique for reducing the overhead by predicting and managing the $\mathrm{MN}$ location adaptively by estimating the mobility pattern was proposed. Similarly, in [29] a method that relies on the data clustering algorithm to estimate the transferred devices' locations was proposed. The device mobility's history is estimated for predicting the future's movements of any particular node. This algorithm attempts to search for appropriate network topology and identify a suitable routing path automatically.

The adaptive estimation related to a particular moving $\mathrm{MN}$ location can only be achieved when the required information's path and speed are existing, and the mobility is taken place at a certain speed on a dedicated path. An adaptive estimation is not applied for the location that belongs to a randomly transferring station. Therefore, before estimating the intended location, the history of mobile activities is exploited for identifying the case of either estimating the location automatically or not. For that reason, adaptive estimation limits the demand for more communications to allocate the transferring device.

An outline summary of this literature is provided in Table II. The direct and indirect techniques and their extensions possess a few drawbacks that are discussed in the literature. In [30] a comparison between the performance of several direct and indirect routing techniques was reported. In [31] and [32] the cost for mobile nodes' mobility throughout 
particular networks was estimated. These studies concluded that an appropriate design of the MIP technique is significant for minimizing the processing and signaling overhead for an updated location.

In conclusion, several methods for minimizing the communication overhead within the mobile IP routing are introduced. Yet, a globally optimized technique for the current internet configuration is required.

TABLE II. COMPARISONS AMONG DIFFERENT MOBILE IP ROUTING ENHANCEMENT METHODS

\begin{tabular}{|c|c|c|c|}
\hline Ref. & Aim & $\begin{array}{l}\text { Briefs of the proposed } \\
\text { methods }\end{array}$ & Drawbacks \\
\hline [19] & $\begin{array}{l}\text { Conceals the } \\
\text { change in mobile } \\
\text { location }\end{array}$ & \multirow{3}{*}{$\begin{array}{l}\text { Distributes HAs to } \\
\text { minimize the distance to } \\
\text { end-node. }\end{array}$} & \multirow{4}{*}{$\begin{array}{l}\text { It is hard to } \\
\text { deploy the } \\
\text { anchors in the } \\
\text { network to avoid } \\
\text { the extra } \\
\text { signaling cost } \\
\text { while avoiding } \\
\text { the HA's } \\
\text { interference. }\end{array}$} \\
\hline$[20]$ & \multirow{3}{*}{$\begin{array}{l}\text { Decentralizes } \\
\text { communication by } \\
\text { deploying agents } \\
\text { instead of the HA }\end{array}$} & & \\
\hline [21] & & & \\
\hline [22] & & $\begin{array}{l}\text { Permits the visiting node } \\
\text { to apply an impermanent } \\
\text { HA within the visited } \\
\text { network }\end{array}$ & \\
\hline [23] & \multirow{4}{*}{$\begin{array}{l}\text { Allows the } \mathrm{MN} \text { to } \\
\text { estimate the } \\
\text { nearest HA }\end{array}$} & $\begin{array}{l}\text { Permits the visiting node } \\
\text { to apply the closest HA } \\
\text { for any casting protocol }\end{array}$ & \multirow{4}{*}{$\begin{array}{l}\text { Required a } \\
\text { history of the } \\
\text { MN and } \\
\text { analyzing such } \\
\text { history }\end{array}$} \\
\hline [24] & & $\begin{array}{l}\text { Relies on a peer-to-peer } \\
\text { HA exploration through } \\
\text { several overlaid peer-to- } \\
\text { peer networks }\end{array}$ & \\
\hline [25] & & $\begin{array}{l}\text { Allows the HA to } \\
\text { preserves the path and } \\
\text { historical information } \\
\text { about the disseminated } \\
\text { HA and MH to search } \\
\text { for an appropriate HA }\end{array}$ & \\
\hline [26] & & $\begin{array}{l}\text { Applies the Markov } \\
\text { procedure, which can } \\
\text { investigate the tracking } \\
\text { cost related to the device } \\
\text { mobility, and which can } \\
\text { search for the most } \\
\text { effective HA }\end{array}$ & \\
\hline [4] & \multirow{2}{*}{$\begin{array}{l}\text { Allows the MN to } \\
\text { work as HA }\end{array}$} & \multirow{2}{*}{$\begin{array}{l}\text { Uses the SDN to enable } \\
\text { each node in the } \\
\text { network to role as an } \\
\text { anchor for the visiting } \\
\text { node }\end{array}$} & \multirow{2}{*}{$\begin{array}{l}\text { Cannot be } \\
\text { generalized in } \\
\text { this form. }\end{array}$} \\
\hline [27] & & & \\
\hline [28] & \multirow{2}{*}{$\begin{array}{l}\text { Estimates of the } \\
\text { location of the } \\
\text { nodes' for cost } \\
\text { minimization }\end{array}$} & $\begin{array}{l}\text { Adaptively predicts the } \\
\text { mobility pattern to } \\
\text { identify a suitable } \\
\text { location }\end{array}$ & \multirow{2}{*}{$\begin{array}{l}\text { Required moving } \\
\text { MN in a specific } \\
\text { pattern }\end{array}$} \\
\hline [29] & & $\begin{array}{l}\text { Estimates the location of } \\
\text { the transferring devices } \\
\text { by applying an analysis } \\
\text { of the network topology }\end{array}$ & \\
\hline
\end{tabular}

\section{PRoposed TeChNiQue}

The proposed semi-direct technique for MIP is based on letting the HA of the local network and the FA of the visiting network to forward the data without encapsulation and decapsulation process. Accordingly, both HA and FA are implementing the routing process besides the address verification process. The $\mathrm{HA}$ is responsible for interacting with a FA of the visiting network to track the CoA of the MN. The $\mathrm{CN}$ request the $\mathrm{CoA}$ of the $\mathrm{HM}$ based on the information stored in the HA. Then, the $\mathrm{CN}$ sends the packet to $\mathrm{MN}$ by using the permanent address and the CoA through the corresponding HA whether a $\mathrm{MN}$ is located in its own home network or a visiting a foreign network. Packets are then forwarded to the FA. The second function of the HA is to verify the addresses associated with the arriving packets, which are addressed to the MNs whose permanent address associated with that HA, but that is currently located in a foreign network. Then, the packets are forwarded from the FA to the MN. The FA also verifies the address as the HA did. In case a changing of the CoA occurred, the information is sent back to $\mathrm{CN}$ in a back-propagation manner. FA informs HA and $\mathrm{HA}$ informs the $\mathrm{CN}$. In turns, the $\mathrm{CN}$ will send a new request to the $\mathrm{HA}$ for the new CoA of the $\mathrm{MN}$. The verification process at the HA depends on the permanent-toCoA mapping addresses that took place at the HA. In the verification at the FA, the process depends on the registration information for the COA only. Accordingly, the proposed technique does not depend on the encapsulation-decapsulating processes, which cause a delay. Thus, this way decreases the delay and increases the speed of transmission. This approach provides a new mechanism for delivering packets to a mobile station. In most cases, the mobile station possesses two IP addresses, which comprise the home address within a home network, which is temporarily connectable through a foreign network when having a foreign agent that uses the CoA. The IP packet consists of a header portion that includes the destination address (CoA).

The packet delivery mechanism of the semi-direct approach is discussed based on three potential scenarios. The first scenario is when the $\mathrm{MN}$ is currently located within the $\mathrm{HN}$. In this manner, the $\mathrm{CN}$ can directly send a packet through to the HA by using different conventional internet routing mechanisms and taking into consideration that the destination IP address of the already sent packet represents permanent address. Because the MN is already located within the HA's scope, it can directly forward the packet through to the intended $\mathrm{MH}$, which receives the packet and replies with a confirmation response should it be required. The second scenario is when the MN is currently located at an FN, and the $\mathrm{CN}$ sends a packet through to the HA where the destination IP address of the sent packet represents the permanent address and the CoA, which forward the packet to the corresponding FA. The third scenario is when the $\mathrm{MN}$ is moving from an FN to another, the $\mathrm{CN}$ sends a packet through to the HA where the destination IP address of the sent packet represents the CoA, the HA verify if there is an update on the destination CoA and, as the HA been informed about the new CoA, the HA response to the $\mathrm{CN}$ with a notification of the changes in the CoA. Accordingly, the $\mathrm{CN}$ sends new request for the new $\mathrm{CoA}$. The HA replies with the new CoA, and the process starts all over.

In Fig. 3, a flowchart of the three potential scenarios about the packet delivery mechanism that is discussed in this section is illustrated. First, in the DA checking, the HA receives the packet and checks its addressing table to identify the current 
temporary IP address (CoA) of the MN. Furthermore, the HA does not perform any encapsulation or header processing. The FA does not implement any processing or decapsulating and thus reduce the processing overhead and the delay. The HA forwards the processed packet through to the FA. When the foreign agent receives the packet, it forwards it directly through to the MN as there is no need for any further header translation. The $\mathrm{MN}$ receives the packet, which contains the $\mathrm{CoA}$ as its destination address, and recognizes that this packet is sent to its visiting $\mathrm{MN}$. If it is required, the mobile station sends a reply message through to the $\mathrm{CN}$.

It should be noted that the source IP address of the reply message represents the MA, and the destination IP address represents the $\mathrm{CN}$. When the $\mathrm{MN}$ is moving from a $\mathrm{FN}$ to another before the $\mathrm{CN}$ get informed and the packet reaches the FA, the FA verify if there is an update on the destination CoA and, as the FA been recognize the movement of the $\mathrm{MN}$, the $\mathrm{FA}$ response to the $\mathrm{CN}$ with a notification of the changes in the $\mathrm{CoA}$. The HA response to the $\mathrm{CN}$ with a notification of the changes as received by the $\mathrm{FA}$. The $\mathrm{CN}$ sends a new request for the new CoA. The HA replies with the new CoA, and the process starts all over.

Mainly, the proposed technique is consists of the following processes: registration, transmission and verification, and notification. These processes are implemented as the $\mathrm{MN}$ move from a network to another away from the MN. As the $\mathrm{MN}$ return to the $\mathrm{HN}$, only the permanent address is used without the need for the transmission of the data.

\section{A. Registration}

In the registration process, FA sends the advertisement message through common protocols, such as Internet Control Message Protocol (ICMP), which are commonly used in diagnostic, error or control purposes. The $\mathrm{MH}$ who just moved to the network within the range of the FA sends a request message for forwarding services to the FA. The FA relays the message to the HA. In case HA accept the request, the HA a registration reply to FA. The FA relays the message to the $\mathrm{MH}$. The HA store the CoA with the permanent address of the $\mathrm{MH}$ as long as the life-time of the registration expires. This process is illustrated in Fig. 4. Both HA and FA maintain the information about mapping between the permanent address and the $\mathrm{CoA}$ for the verification purposes. As a new request is received for $\mathrm{CoA}$ of registered $\mathrm{MH}$, the old value is replaced, and the new and old value shall be used for the verification of the transmitted data.

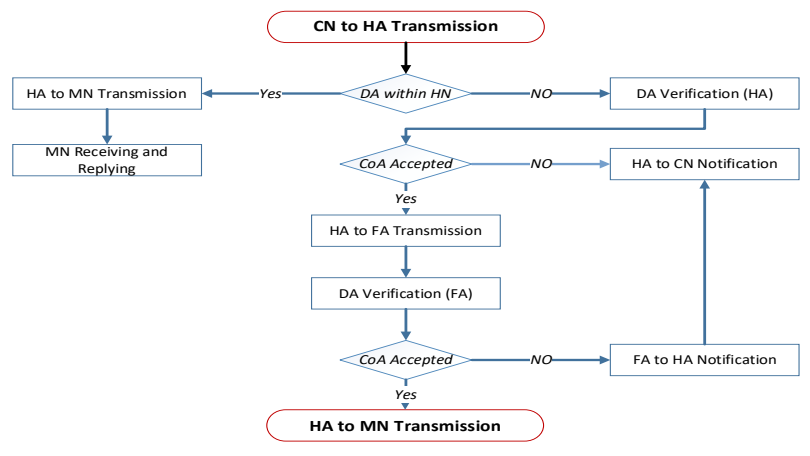

Fig. 3. Flowchart of the Proposed Technique.

\section{B. Transmission and Verification}

Datagram transmission is implemented in a sequence if the $\mathrm{MH}$ is located in $\mathrm{FN}$, and the registration has been implemented successfully. In such a case, the $\mathrm{MH}$ send the datagram to the HA, which will verify the DA and relay the transmitted packet to the FA, which in turn verify the DA and relay it to the $\mathrm{MH}$. In the verification process, HA stores information about the MA and the CoA of the MH. Similarly, the FA maintain such information to implement his identification purpose. Accordingly, both agents verify the transmission by checking the DA (see Fig. 3).

\section{Notification}

In the notification process, FA and $\mathrm{HA}$ send the notification through common protocols, such as ICMP, when there are modification on the records concerned the $\mathrm{MH}$. In case that the $\mathrm{MH}$ get away from the visiting network, the FA sends a notification message for error in the DA. The HA relays the message to the $\mathrm{CN}$. In case that $\mathrm{HA}$ recognize such error in the verification process, the notification is sent back to the $\mathrm{CN}$ (see Fig. 5). In such a case, the $\mathrm{CN}$ send a request for CoA for the HA and start the transmission back when a reply is received for the new CoA.

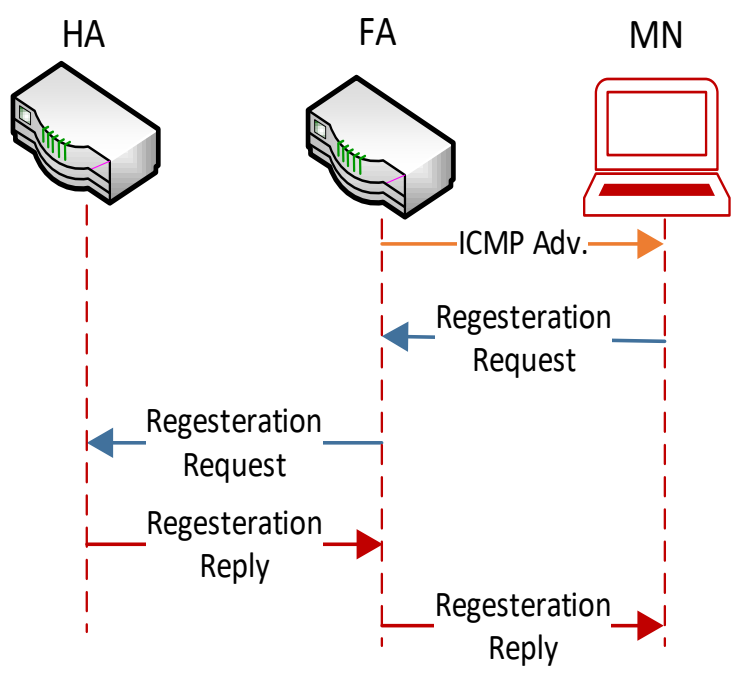

Fig. 4. Registration Sequence.

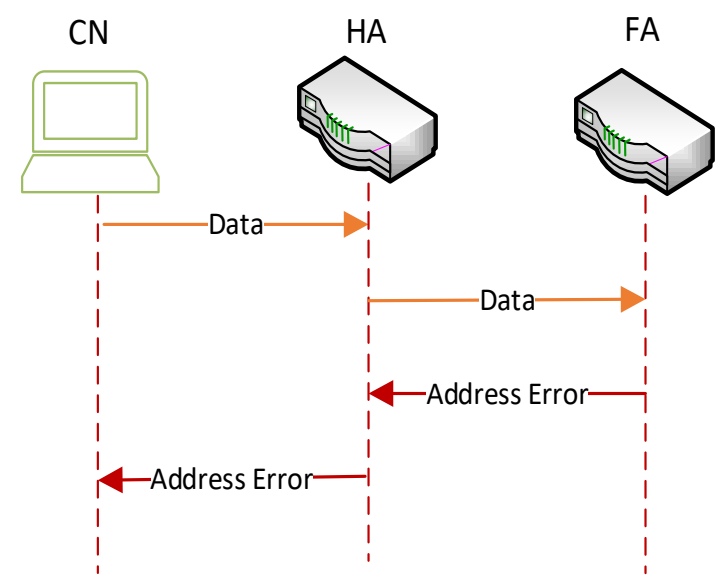

Fig. 5. Notification Sequence. 


\section{Comparison}

Accordingly, the proposed technique is semi-direct in as the HA and the FA intermediate the transmission process for validating processes, yet, there is no header processing nor encapsulating and decapsulating processes. Accordingly, as similar to the indirect technique, the proposed approach keeps track of the $\mathrm{MH}$ movement through the verification and updating processes and as similar to the direct technique it minimizes the transmission processes by avoiding the encapsulating and decapsulating processes. The disadvantage of the proposed approach, the location of the $\mathrm{MH}$ is not transparent to the $\mathrm{CN}$. A comparison between the proposed and the indirect and direct techniques are given in Table III.

TABLE III. COMPARISONS OF THE PROPOSED TECHNIQUE AND THE EXISTING TECHNIQUES

\begin{tabular}{|l|l|l|l|}
\hline Indirect Approach & Direct Approach & $\begin{array}{l}\text { Semi-Direct } \\
\text { Approach }\end{array}$ \\
\hline HA & $\begin{array}{l}\text { Receives all the } \\
\text { transmitted packets, } \\
\text { implements } \\
\text { encapsulation and } \\
\text { transmission to FA }\end{array}$ & $\begin{array}{l}\text { Receives request for } \\
\text { the CoA and replies } \\
\text { based on the stored } \\
\text { information. }\end{array}$ & $\begin{array}{l}\text { Receives request for } \\
\text { CN CoA, replies to } \\
\text { stored information, } \\
\text { verifies CoA, } \\
\text { receives notification } \\
\text { of changes from FA } \\
\text { and notifies CN. }\end{array}$ \\
\hline $\begin{array}{l}\text { Receives all the } \\
\text { transmitted } \\
\text { encapsulated packets, } \\
\text { decapsulates them and } \\
\text { transfers them through } \\
\text { to the visiting MH }\end{array}$ & $\begin{array}{l}\text { Registers CoA of } \\
\text { the visiting MH }\end{array}$ & $\begin{array}{l}\text { Registers and } \\
\text { verifies CoA, and } \\
\text { notifies HA with } \\
\text { CoA changes }\end{array}$ \\
\hline MH & $\begin{array}{l}\text { Registers the CoA } \\
\text { with the FA }\end{array}$ & $\begin{array}{l}\text { Registers the CoA } \\
\text { with the FA }\end{array}$ & $\begin{array}{l}\text { Registers the CoA } \\
\text { with the FA }\end{array}$ \\
\hline CN & $\begin{array}{l}\text { Sends all the } \\
\text { transmitted packets to } \\
\text { the HA }\end{array}$ & $\begin{array}{l}\text { Sends all the } \\
\text { transmitted packets } \\
\text { to the MH }\end{array}$ & $\begin{array}{l}\text { Sends all the } \\
\text { transmitted packets } \\
\text { to the HA }\end{array}$ \\
\hline
\end{tabular}

\section{ANALYSIS, EXPERIMENTS AND RESUlTS}

In order to analyze the performance of the proposed technique and the existing techniques in term of the processing overhead and the speed of the transition, delay is used for comparison purpose. Based on the discussed processed for these techniques, there are differences in the total delay based on the location of the $\mathrm{MH}$, and there is different processing that required and causes such delay. The delays that are caused by the discussed techniques is registration delay (DR), encapsulation/decapsulation delay (DCap) and Transmission delay (DT). A numerical analysis and simulation are carried out to provide relevant comparisons among the evaluate techniques. Accordingly, all the scenarios of the connection and transmission between the $\mathrm{CN}$ and the $\mathrm{MH}$ are analyzed and the total delay at each scenario is calculated. In the numerical analysis, without loss of generality, we assume that the transmission delay is identical regardless of the identity of the sender and the receiver nodes. Accordingly, we also assume that the transmission delay and the CoA registration are identical. Three scenarios depicted these are, $\mathrm{MH}$ within the home network (S1), $\mathrm{MH}$ is in an $\mathrm{FN}$ (S2), and $\mathrm{MH}$ moved from FN to another with probability 0.5 (S3). Accordingly, the delay of each of the depicted scenario is calculated as given in Table IV.
TABLE IV. TOTAL DELAY IN THE DiRECT APPROACH AND Follow ME APPROACH

\begin{tabular}{|c|c|c|c|c|c|c|}
\hline \multirow[b]{2}{*}{ \# } & \multicolumn{2}{|l|}{ Direct } & \multicolumn{2}{|l|}{ Indirect } & \multicolumn{2}{|l|}{ Semi-Direct } \\
\hline & Equation & Unity & Equation & Unity & Equation & Unity \\
\hline S1 & $D_{\text {total }}=D_{T}$ & 1 & $\begin{array}{l}D_{\text {total }} \\
=D_{T}\end{array}$ & 1 & $D_{\text {total }}=D_{T}$ & 1 \\
\hline S2 & $\begin{array}{l}D_{\text {total }} \\
=D_{R}+D_{T}\end{array}$ & 2 & $\begin{array}{l}D_{\text {total }} \\
=D_{R}+3 \\
* D_{T} \\
+D_{\text {Cap }}\end{array}$ & 5 & $\begin{array}{l}D_{\text {total }} \\
=D_{R}+3 \\
* D_{T}\end{array}$ & 4 \\
\hline S3 & $\begin{array}{l}D_{\text {total }} \\
=\sum_{i=1}^{n} D_{R} \\
+D_{T}\end{array}$ & $2 n$ & $\begin{array}{l}D_{\text {total }} \\
=D_{R}+3 \\
* D_{T} \\
+D_{\text {Cap }}\end{array}$ & 5 & $\begin{array}{l}D_{\text {total }} \\
=D_{R}+3 \\
* D_{T}\end{array}$ & 4 \\
\hline
\end{tabular}

Accordingly, the direct technique is good for $\mathrm{MH}$ that is not frequently moving through the network, which is not the case in real implementation. Thus, the proposed technique is best compared to the indirect approach. A comparison between the proposed technique and the indirect technique are given in Fig. 6, Fig. 7 and Fig. 8. If five packets with the following payloads (Bytes): 5000, 10000, 15000, 20000 and 25000, are sent from the Correspondent Network (CN) along to a Mobile Station (MS), the results of each scenario are illustrated.

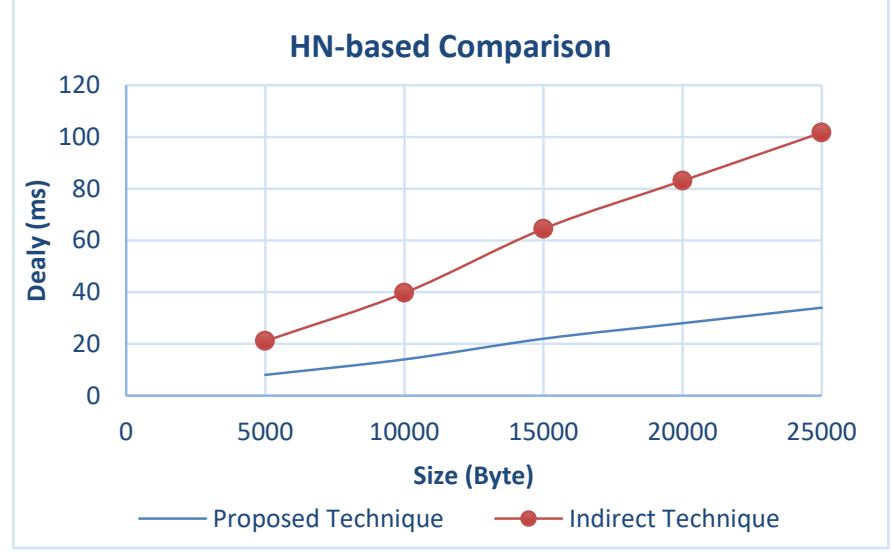

Fig. 6. A Comparison between the Propose and the Indirect Techniques for MH Located within the HN.

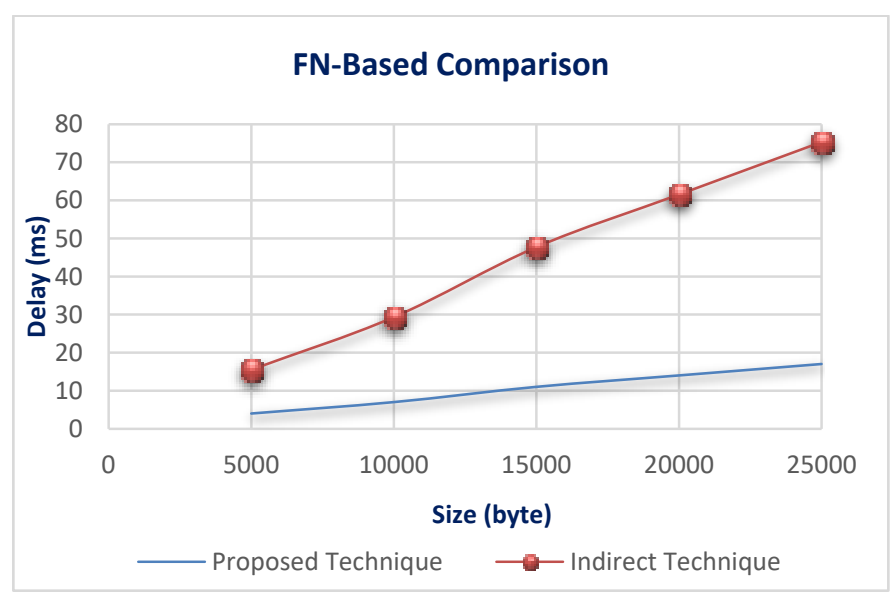

Fig. 7. A Comparison between the Propose and the Indirect Techniques for MH Located in an FN. 


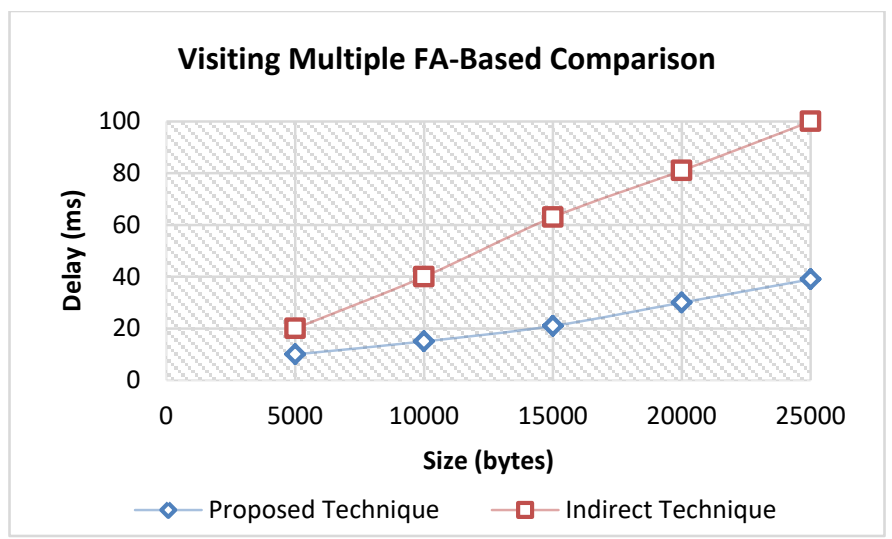

Fig. 8. A Comparison between the Propose and the Indirect Techniques for MH Moving from FA to another.

Based on the previously obtained results, it can be inferred that the delivery of data when using the semi-direct technique for mobile IP is found faster than the delivery of data when using the indirect technique. The reason behind this is that some processes as the encapsulation/decapsulation and additional fragmentation/de-fragmentation are only implemented in the indirect technique of the mobile IP routing technique, and is not implemented in the proposed technique. Thus, any other process requires an additional time. The total checksum time delay is found larger in the conventional indirect routing technique than in the proposed technique due to the fragmentation process in the HA, which uses a conventional approach. This implies that each fragment requires a checksum process. If the $\mathrm{HA}$ that uses the encapsulation of the mobile IP receives $\mathrm{N}$ packets, the number of output packets represents $2 \mathrm{~N}$ or $2 \mathrm{~N}-1$ packets. The obtained results prove the correctness of the conclusion mentioned above.

\section{CONCLUSION}

Recently, the encapsulation of packets is the well-known approach of the Mobile IP indirect routing approach, which is still uncommon for using the non-encapsulation approach with the mobile IP indirect routing technique. In this paper, it is inferred from the simulation results for implementing the nonencapsulation approach is found more efficient in comparison with the conventional encapsulation approach. Additionally, the encapsulation process itself increases the transmission delay, and the process of encapsulation for sending packets can enlarge their sizes. Furthermore, the speed of the communication process can be decreased due to the enlarged size of the new packet that should be sent from the home agent's side. Moreover, the de-encapsulation process requires an additional time to return the new packet through to its original structure so that this process can increase the total delay of the communication process. The future work will focus on implementing the semi-direct technique with multiple anchor distribution to further reduce the delay and signalling cost.

\section{REFERENCES}

[1] Z. Zhu, R. Wakikawa, and L. Zhang, "A survey of mobility support in the Internet”, IETF Request for Comments, 2011, 6301.
[2] C. Perkins, "IP mobility support for IPv4, revised", ternet Engineering Steering Group, 2010.

[3] C. Perkins, D. Johnson, and J. Arkko, "RFC 6275 Mobility Support in IPv6", Internet Engineering Task Force (IETF), 2011.

[4] Y. Wang, and J. Bi, "Software-defined mobility support in IP networks", The Computer Journal, vol. 59 (2), pp. 159-177, 2016.

[5] J.M. Opos, S. Ramabhadran, A. Terry, J. Pasquale, A.C. Snoeren, and A. Vahdat, "A performance analysis of indirect routing", in Book A performance analysis of indirect routing' (IEEE, 2007, edn.), pp. 1-10.

[6] P. Pupatwibul, A. Banjar, A.A. Sabbagh, and R. Braun, "Developing an application based on openflow to enhance mobile ip networks", in "Book Developing an application based on openflow to enhance mobile ip networks" (IEEE, 2013, edn.), pp. 936-940.

[7] A. Boukerche, A. Magnano, and N. Aljeri, "Mobile IP handover for vehicular networks: Methods, models, and classifications", ACM Computing Surveys, CSUR, vol. 49 (4), pp. 1-34, 2017.

[8] M.S. Chiang, and C.M. Huang, "A backward fast handover control scheme for mobile internet (BFH-MIPv6)", Journal of Internet Technology, vol. 19 (2), pp. 359-367, 2018.

[9] M.H. Rahman, N. Islam, A.I. Swapna, and M.A. Habib, "Analysis of Software Defined Wireless Network with IP Mobility in Multiple Controllers Domain", in Book Analysis of Software Defined Wireless Network with IP Mobility in Multiple Controllers Domain, (Springer, 2020, edn.), pp. 529-538.

[10] C.E. Perkins, "Mobile networking through mobile IP", IEEE Internet computing, vol. 2 (1), pp. 58-69, 1998.

[11] J.H. Lee, J.M. Bonnin, and X. Lagrange, "Host-based distributed mobility management support protocol for IPv6 mobile networks", in Book Host-based distributed mobility management support protocol for IPv6 mobile networks, (IEEE, 2012, edn.), pp. 61-68.

[12] C. Anastasiades, T. Braun, and V.A. Siris, "Information-centric networking in mobile and opportunistic networks", in Wireless Networking for Moving Objects (Springer, 2014), pp. 14-30.

[13] J. Lee, and S. Pack, "Mobility management in future wireless networks: Past, present, and future", IEEE WCNC Tutorial, 2013.

[14] A. Udugama, M.U. Iqbal, U. Toseef, C. Goerg, C. Fan, and M. Schlaeger, "Evaluation of a network based mobility management protocol: PMIPv6", in Book Evaluation of a network based mobility management protocol: PMIPv6, (IEEE, 2009, edn.), pp. 1-5.

[15] T. Clausen, U. Herberg, and J. Yi, "Security Threats to the Optimized Link State Routing Protocol Version 2 (OLSRv2)", in Book Security Threats to the Optimized Link State Routing Protocol, Version 2 (OLSRv2)' (2017, edn.),

[16] C. Li, M.K. Mukerjee, D.G. Andersen, S. Seshan, M. Kaminsky, G. Porter, and A.C. Snoeren, "Using indirect routing to recover from network traffic scheduling estimation error", in Book Using indirect routing to recover from network traffic scheduling estimation error, (IEEE, 2017, edn.), pp. 13-24.

[17] S.H. La, J. Jeong, J. Koo, and U.M. Kim, "On intelligent hierarchical FPMIPv6 based mobility support for industrial mobile networks", Procedia Computer Science, vol. 155, pp. 169-176, 2019.

[18] I. Al-Surmi, M. Othman, and B.M. Ali, "Mobility management for IPbased next generation mobile networks: Review, challenge and perspective", Journal of Network and computer Applications, vol. 35(1), pp. 295-315, 2012.

[19] R. Wakikawa, G. Valadon, and J. Murai, "Migrating home agents towards internet-scale mobility deployments", in Book Migrating home agents towards internet-scale mobility deployments (2006, edn.), pp. 110.

[20] Y. Mao, B. Knutsson, H. Lu, and J.M. Smith, "Dharma: Distributed home agent for robust mobile access", in Book Dharma: Distributed home agent for robust mobile access, (IEEE, 2005, edn.), pp. 11961206.

[21] Y. Wang, J. Bi, and X. Jiang, "A multi - anchoring approach in mobile IP networks", Wireless Communications and Mobile Computing, vol. 16 (18), pp. 3423-3438, 2016. 
[22] R. Zheng, Y. Ge, J.C. Hou, and S.R. Thuel, "A case for mobility support with temporary home agents", ACM SIGMOBILE Mobile Computing and Communications Review, vol. 6 (1), pp. 32-46,2002.

[23] Y.S. Yen, C.C. Hsu, Y. Chan, and H. Chao, "Global dynamic home agent discovery on mobile IPv6", 2nd International Conference on Mobile Technology, Applications and Systems, November 2005.

[24] R. Cuevas, C. Guerrero, A. Cuevas, M. Calderon, and C.J. Bernardos, "P2P based architecture for global home agent dynamic discovery in IP mobility", in Book P2P based architecture for global home agent dynamic discovery in IP mobility, (IEEE, 2007, edn.), pp. 899-903.

[25] G. Motoyoshi, K. Leibnitz, and M. Murata, "Proposal and evaluation of a function-distributed mobility architecture for the future internet", IEICE transactions on communications, vol. 94 (7), pp. 1952-1963, 2011.

[26] K.T. Chen, S.L. Su, and R.F. Chang, "Design and analysis of dynamic mobility tracking in wireless personal communication networks", IEEE Transactions on Vehicular Technology, vol. 51 (3), pp. 486-497, 2002.

[27] A. Bradai, A. Benslimane, and K.D. Singh, "Dynamic anchor points selection for mobility management in Software Defined Networks", Journal of Network and Computer Applications, vol. 57, pp. 1-11, 2015.
[28] W.J. Choi, and S. Tekinay, "An adaptive location registration scheme with dynamic mobility classification", in Book An adaptive location registration scheme with dynamic mobility classification, (IEEE, 2002, edn.), pp. 440-444.

[29] J. Taheri, A.Y. and Zomaya, "Clustering techniques for dynamic location management in mobile computing", Journal of Parallel and Distributed Computing, vol. 67(4), pp. 430-447, 2007.

[30] V.S. Wong, V.C. and Leung, "Location management for next-generation personal communications networks", IEEE network, 14 (5), pp. 18-24, 2000.

[31] J.S. Ho, I.F. and Akyildiz, "A dynamic mobility tracking policy for wireless personal communications networks', in Book A dynamic mobility tracking policy for wireless personal communications networks, (IEEE, 1995, edn.), pp. 1-5.

[32] J. Li, H. Kameda, and K. Li, "Optimal dynamic mobility management for PCS networks", IEEE/ACM Transactions on Networking, vol. 8(3), pp. 319-327, 2000. 